\title{
Worldwide Perspectives of Amyloidosis
}

\author{
Iuliana Vaxman ${ }^{\mathrm{a}-\mathrm{c}}$ Morie A. Gertz \\ ${ }^{a}$ Division of Hematology, Mayo Clinic, Rochester, MN, USA; ${ }^{b}$ Institute of Hematology, Davidoff Cancer Center, \\ Rabin Medical Center, Petah-Tikva, Israel; ' Sackler Faculty of Medicine, Tel-Aviv University, Tel-Aviv, Israel
}

Amyloidosis is a rare and heterogeneous group of disorders that are characterized by extracellular deposition of abnormally folded proteins in tissues, ultimately leading to organ dysfunction. The prognosis is grave if it is detected in late stages, especially with extensive cardiac involvement. Progress has been made in the last decade in all aspects of amyloidosis. In this special edition of Acta Haematologica, experts from all over the world contribute their knowledge and experience in various aspects of amyloidosis.

Early diagnosis of amyloidosis continues to pose a significant challenge and requires a high index of suspicion and the collaboration of many disciplines (internists, primary care providers, cardiologists, nephrologists, neurologists, and hematologists). The review "When to suspect amyloid?" [1] emphasizes the warning signs that should alert a physician evaluating a patient. Hematologists following monoclonal gammopathy of undetermined significance and smoldering multiple myeloma should be alert to the possibility of the development of amyloidosis even if light chains are not rising and they must monitor clinical signs and symptoms, 24-h urinary protein, alkaline phosphatase, and cardiac biomarkers.

An accurate diagnosis of amyloidosis is important and, in an excellent review on confirming the diagnosis of amyloidosis, Wisniowski and Wechalekar [2] emphasizes the importance of attaining a histological specimen from an affected organ or a surrogate site (bone marrow, abdominal fat pad, or salivary gland) and the specific considerations in choosing the appropriate site to biopsy. The exception is cardiac ATTR amyloidosis, in which a nonbiopsy diagnosis can be confirmed in the absence of any evidence of a monoclonal light chain. Wisniowski and Wechalekar [2] provides a useful algorithm for confirming the diagnosis of AL amyloidosis.

While there is some predilection of certain amyloid types for particular organs, the determination of the amyloid type on clinical grounds alone is not reliable and typing of the amyloid protein is imperative for accurately diagnosing a patient. In her comprehensive review about the pathology of amyloidosis, Picken [3] underlines the importance of differentiating localized from systemic amyloidosis and delves into the different typing methods and their limitations, i.e., immune methods (immunofluorescence, immunohistochemistry, and immunogold labelling) and proteomic methods (mass spectrometry). While mass spectrometry has emerged as the preferred method of amyloid typing, it is not widely available and careful application of immune methods is still clinically useful in experienced centers. karger@karger.com www.karger.com/aha

(c) 2020 S. Karger AG, Basel

Karger"
Morie A. Gertz

Mayo Clinic 200 First St. SW

Rochester, MN 55905 (USA)

gertz.morie@mayo.edu 
Fig. 1. The Mayo Clinic's treatment algorithm for newly diagnosed AL amyloidosis patients who are transplant ineligible (consider adding doxycycline for at least a year). BMPC, bone marrow plasma cells; CyBorD, cyclophosphamide, bortezomib, and dexamethasone; VGPR, very good partial response. ${ }^{1}$ Or BMel-Dex. ${ }^{2}$ If $<$ partial response at 2 months, consider changing the therapy. ${ }^{3}$ If the patient is young, consider stem cell collection for eventual autologous stem cell transplantation. ${ }^{4}$ Make the decision to change/add therapy if there is a complete response based on a number of clinical factors. Rerefer to an amyloid center of excellence.

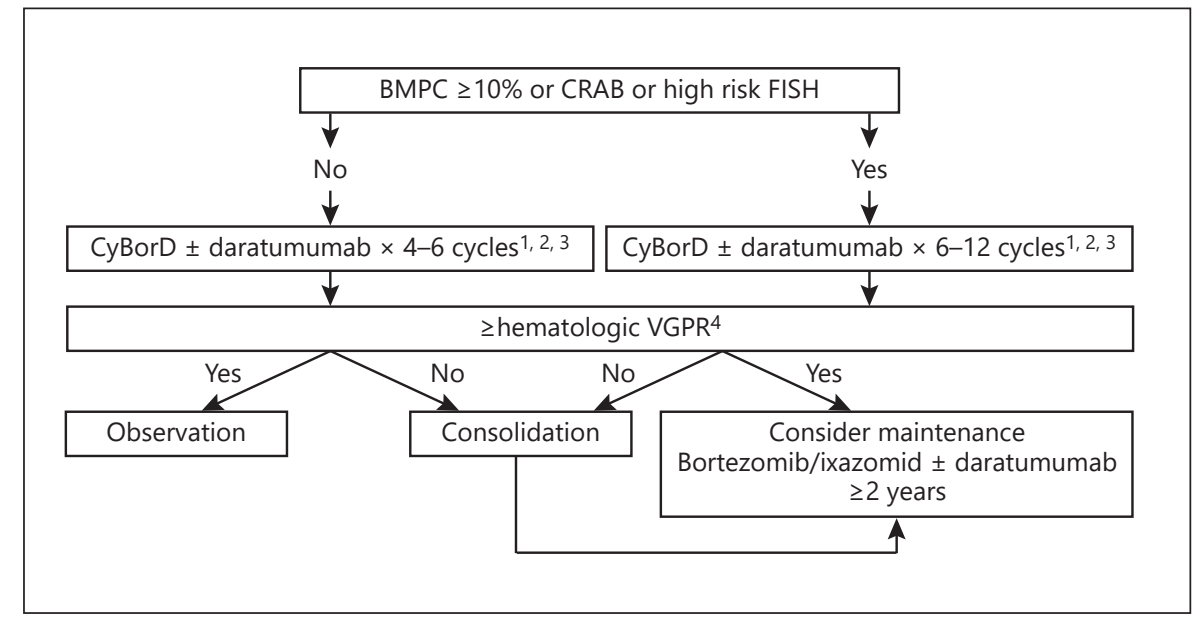

Management of AL amyloidosis should be multidisciplinary, involving experts in hematology, cardiology, nephrology, gastroenterology, and neurology. Treatment has two major components, i.e., supportive care and antiplasma cell-directed therapy. In their remarkable review, Cibeira et al. [4] address the important issue of supportive care, aimed at maintaining quality of life and preserving organ function.

Diuretics are the mainstay of therapy for cardiac and renal amyloidosis, helping to reduce edema, but they can induce hypotension and creatinine elevation. Avoiding hypotension, infections, and contrast media in order to preserve renal function is imperative. $\beta$-Blockers have a negative inotropic and chronotropic effect and may lead to a reduced cardiac output and cause hypotension.

Zadok and Kornowski [5] provide a comprehensive review from a cardiology standpoint concerning amyloid-specific treatment strategies. They suggest limiting the use of $\beta$-blockers to selected patients with atrial fibrillation and a rapid ventricular rate nonresponsive to other rate control measures. The authors provide a practical table with the essentials of managing common cardiac issues in cardiac amyloidosis.

Outcomes of solid organ transplantation are improving and Theodorakakou et al. [6] share their knowledge in this field in the different subtypes of amyloid, emphasizing the gap in knowledge about the timing of the transplant and the need to establish eligibility criteria. The available data are mostly retrospective and include relatively small numbers of patients. The main concerns with renal, cardiac, and liver transplantation are acute rejection, amyloidosis recurrence that limits the long-term graft survival, and organ donor shortages. Advances in supportive care and a better ability to achieve deep responses make solid organ transplantation a treatment modality that should be considered in appropriately selected patients. Theodorakakou et al. [6] point out the need for an international collaboration to define selection criteria and the timing of transplantation.

Prompt treatment directed at the underlying plasma cell clone is crucial, aiming to achieve a deep and rapid response and therefore improving quality of life and survival. Figure 1 shows the Mayo Clinic recommendations for treating transplant-ineligable patients. Anti-plasma cell-directed therapy is comprised of nonchemotherapy treatment, conventional chemotherapy, and autologous stem cell transplantation. Milani and Palladini [7] supplies us with a very well-organized review on the value of conventional therapy in amyloidosis. The readers will also enjoy the review of Van Doren and Lentzsch [8], which leads us through nonchemotherapy treatments of amyloidosis and presents data on the current standard of care, VCd, as well as emerging evidence of the important role of daratumumab [9-12], which may define the new standard of care [13]. Sanchorawala [14], who arguably have the world's greatest experience, provide a comprehensive review about the timing and role of autologous stem cell transplantation in amyloidosis, as well as selection criteria.

In spite of all of the advances made in the accurate diagnosis and treatment of amyloid patients, the disease remains fatal as organ failure is often irreversible if the diagnosis is made at an advanced stage. The prognosis of $\mathrm{AL}$ patients is determined by the biology of the underlying clonal disease, the pattern and extent of organ involvement at baseline, and the response to treatment. New systems for 
assessing the prognosis and new staging systems are developing rapidly and, in their informative review, Dittrich et al. [15] review the available data about prognostic factors and staging systems, summarizing the data in useful tables.

We hope that you will find this special edition enriching, practical, and exciting. Through this issue the following key points are emphasized: (1) the importance of establishing an early diagnosis by considering amyloidosis in all patients with proteinuria, heart failure with a preserved ejection fraction, unexplained hepatomegaly, and peripheral or autonomic neuropathy; (2) the essential role of typing of the amyloid and determining which organs are involved in order to obtain an accurate diagnosis and develop a treatment plan; and (3) recognition of the introduction of daratumumab in the treatment of AL amyloidosis as an important next step in improving survivorship in this serious disorder.

\section{Conflict of Interest Statement}

M.A. Gertz reports personal fees from: Ionis/Akcea; Alnylam; Prothena; Janssen; Annexon; Appellis; Amgen; Medscape; Physicians Education Resource; the Data Safety Monitoring Board of Abbvie and Celgene; Research to Practice, and Workforce Training Sanofi. M.A. Gertz also reports grants and personal fees from Spectrum, speaker fees from Teva, Johnson and Johnson, Medscape, and DAVA oncology. M.A. Gertz is on the Pharmacyclics Advisory Board for Proclara, has participated in the development of educational materials and programs for i3Health, has received royalties from Springer Publishing, and has stock options in Aurora Bio.

\section{Funding Sources}

Grant funding was received from the Amyloidosis Foundation and the International Waldenstom Foundation (NCI SPORE MM SPORE 5P50 CA186781-04).

\section{References}

1 Vaxman I, Gertz M. When to Suspect a Diagnosis of Amyloidosis. Acta Haematol. 2020 Apr 27:1-8.

2 Wisniowski B, Wechalekar A. Confirming a Diagnosis of Amyloidosis. Acta Haematol. 2020. DOI: $10.1159 / 000508022$.

3 Picken MM. The Pathology of Amyloidosis in Classification: A Review. Acta Haematol. 2020 May; $1-13$.

4 Cibeira MT, Ortiz-Pérez JT, Quintana LF, Fernadez de Larrea C, Tovar N, Bladé J. Supportive Care in AL Amyloidosis. Acta Haematol. 2020. DOI: 10.1159/000506760.

5 Zadok OIB, Kornowski R. Cardiac Care of Patients with Cardiac Amyloidosis. Acta Haematol. 2020. DOI: 10.1159/000506919.

6 Theodorakakou F, Fotiu D, Dimopoulos MA, Kastritis E: Solid Organ Transplantation in Amyloidosis. Acta Harmatol. 2020. DOI: 10.1159/000508262.

7 Milani P, Palladini G. Conventional Therapy for Light-Chain Amyloidosis. Acta Haematol. 2020. DOI: $10.1159 / 000507072$.
8 Van Doren L, Lentzsch S. Nonchemotherapy Treatment of Immunoglobulin Light Chain Amyloidosis. Acta Haematol. 2020. DOI: 10.1159/000507724.

9 Cohen OC, Brodermann MH, Blakeney IJ, Mahmood S, Sachchithanantham S, Ravichandran S, et al. Rapid response to single agent daratumumab is associated with improved progression-free survival in relapsed/ refractory AL amyloidosis. Amyloid. 2020:16.

10 Milani P, Fazio F, Basset M, Berno T, Larocca A, Foli A, et al. High rate of profound clonal and renal responses with daratumumab treatment in heavily pre-treated patients with light chain (AL) amyloidosis and high bone marrow plasma cell infiltrate. Am J Hematol. 2020 Apr;ajh.25828.

11 Roussel M, Merlini G, Chevret S, Arnulf B, Stoppa AM, Perrot A, et al. A prospective phase 2 trial of daratumumab in patients with previously treated systemic light-chain amyloidosis. Blood. 2020 Apr;135(18):1531-40.
12 Sanchorawala V, Sarosiek S, Schulman A, Mistark M, Migre ME, Cruz R, et al. Safety, tolerability, and response rates of daratumumab in relapsed AL amyloidosis: results of a phase 2 study. Blood. 2020 Apr;135(18): 1541-7.

13 Palladini G, Kastritis E, Maurer MS, Zonder JA, Minnema MC, Wechalekar AD, et al. Daratumumab Plus CyBorD for Patients With Newly Diagnosed AL Amyloidosis: Safety Run-in Results of ANDROMEDA. Blood. 2020 Apr;blood.2019004460.

14 Sanchorawala V. High-Dose Melphalan and Autologous Peripheral Blood Stem Cell Transplantation in AL Amyloidosis. Acta Haematol. 2020. DOI: 10.1159/000506498.

15 Dittrich T, Kimmich C, Hegenbart U, Schönland SO. Prognosis and Staging of AL Amyloidosis. Acta Haematol. 2020. DOI: $10.1159 / 000508287$. 